Journal of Applied Fluid Mechanics, Vol. 4, No. 1, pp. 77-88, 2011.

Available online at www.jafmonline.net, ISSN 1735-3572, EISSN 1735-3645.

DOI: $10.36884 / \mathrm{jafm} .4 .01 .11904$

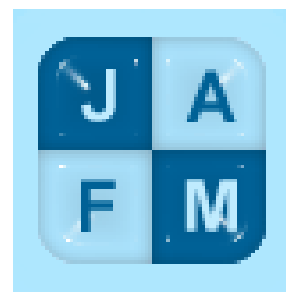

\title{
Influence of Falling Height on the Behavior of Skid- Launching Free-Fall Lifeboat in Regular Waves
}

\author{
M. M. Karim ${ }^{1}$, K. S. Iqbal ${ }^{1}$, M. R. H. Khondoker ${ }^{1}$ and S. M. H. Rahman ${ }^{2}$ \\ ${ }^{I}$ Dept. of Naval Architecture and Marine Engineering, Bangladesh University of Engineering and Technology, \\ Dhaka-1000, Bangladesh \\ ${ }^{2}$ American Bureau of Shipping (ABS)
}

Email:mmkarim@name.buet.ac.bd

(Received August 14, 2008; accepted October 28, 2009)

\begin{abstract}
This paper numerically investigated the influence of falling height on the behavior of the skid-launching free-fall lifeboat (FFLB) in regular waves. The boat has been treated as a rigid body when the differential equations of motion for the four falling phases, i.e., sliding or ramp phase, rotation phase, free-fall phase and water entry phase of the lifeboat were solved in the time domain. The hydrodynamic characteristics of the lifeboat has been studied for different falling heights such as $H=1.5 \mathrm{~m}, 1.75 \mathrm{~m}$ and $2.00 \mathrm{~m}$. Horizontal and vertical excursions and the rotation of the axis of the boat have been computed at different time along with its horizontal and vertical velocities. Hydrodynamic forces and accelerations at normal and axial directions have also been determined. At first the analysis has been done in still water and then in regular wave with amplitude of $0.5 \mathrm{~m}$ and a period of $2.0 \mathrm{sec}$. In all of the cases, effects of regular wave are shown by comparing the results with those considering the falling of FFLB into calm water.
\end{abstract}

Keywords: Hydrodynamics, Numerical simulation, Motion characteristics, Acceleration, Added mass, Momentum transfer.

\section{INTRODUCTION}

Shipping industries almost all over the world are now showing very much interest on free-fall lifeboat as many life threatening accidents have occurred with conventional lifeboat systems in the past. Most of the accidents happened during launching and after lowering the boat into rough seas in high wind. During launch, the lifeboat may hit the sides of the distressed vessel, become severely damaged and occupants may fall into the sea causing injury and even death. It is impossible to launch the lifeboat if the parent vessel is listing significantly or if the falling becomes tangled. After lowering the boat into the water, it may be unable to move away from the distressed vessel if high seas and winds continually push the lifeboat towards the parent vessels or due to the inability of the engine to start. These situations become even more dangerous during fire or when the potential for an explosion exists.

A lot of risks associated with conventional lifeboat systems have been substantially reduced by the free-fall lifeboat system. These problems are minimized with the free-fall lifeboat because it is not lowered into the sea. The free-fall lifeboat falls freely into the sea, generating kinetic energy which propels the lifeboat away from the distressed vessel during and immediately after water entry. The lifeboat moves away from the danger even if the engine does not operate.

Many researchers made investigations on freely falling object. Aanesland (1987) studied the dynamic motion of freely falling drilling pipe in water by using slender body theory. The coupled surge-heave-pitch motion of the cylinder, in the formulation, correspond to the coupled surge-sway-yaw motion of a ship based on the maneuvering equations as presented by Newman (1977) corrected by viscous effects. The most important effect of water impact when penetrating through the free water surface has not been included in the formulation.

Boef (1992) proposes a practical method for simulating the water entry of a free-fall lifeboat. The literature on the hydrodynamic impact is reviewed and the concepts of momentum balance and added mass are explored by analyzing the impact of various cylinders. The theoretical model for the water entry of a lifeboat is developed by extending the concepts cylinder impact and seaplane landing. The second part of his study discusses the implementation of the lifeboat launch model and a method for evaluating the effects of acceleration on the occupants based on Dynamic Response Model of Brinkley et al. (1971). 
Luo and Davis (1992) studied the free-fall motion of the dropped objects and their impact on underwater installations. They formulated the equations of motion in global coordinate system transferring the normal and tangential drag force from local coordinate system.

Colwill and Ahilan (1992) used the same mathematical approach developed by Luo and Davis (1992) and conducted a reliability analysis of the behavior of dropped objects. At first the experimental verification of the numerical method has been done using Aanesland's experimental result.

Campbell and Claughton (1983) presented the experimental work on the alternative means of launching lifeboats from offshore rigs and mobiles. They have conducted test in wind and wave over the conventional twin-fall system, an outward facing single fall and tug line system, a boom launch system and a radical free-fall lifeboat system. They have discussed the difficulties of successfully launching lifeboats from the windward or up wave side of a rig in storm conditions.

Muler and Payer (1983) studied on the estimation of load and strength of free-fall lifeboats. Very simple approximation has been done for calculating the hydrodynamic slamming pressure and the boat has been discretized and analyzed by finite element method. Experimental investigation has also been conducted to verify the acceleration in different positions.

Nelson et al. (1991) presented a mathematical model to predict the launch behavior of free-fall lifeboat considering the boat as an assemblage of triangular plates. In another research, Nelson et al. (1994) described the implementation of free-fall lifeboats in ships, the relative economics of free-fall lifeboat systems compared with davit launched lifeboat systems and anticipated improvements in safety afforded by free-fall lifeboats during an emergency. The other study by Nelson et al. (1995) discussed quantitatively the launch behavior of free-fall lifeboats with particular emphasis on the effects of changes in the location of CG.

Tasaki et al. (1990) described a numerical simulation method on the falling motion of free-fall lifeboat. After validating the method through experiment, the relation between the altitude and launching conditions has been discussed.

Arai et al. (1994, 1995, 1996a, 1996b, 1996c) developed a numerical model for the simulation of motion and behaviors of the realistic lifeboat for the first time. The effect of acceleration on the occupant of a lifeboat and the elevation of effective launching parameters of a freefall lifeboat has also been studied by them. However, they did not include the effect of wave in the model. In this present study, the same model is extended to analysis motion and acceleration of a free-fall lifeboat falling into the regular waves and influence of falling height on its behaviour is investigated.

\section{MATHEMATiCAL Formulation}

\subsection{Launch Simulation of a Free-fall Lifeboat}

The configuration of a free-fall lifeboat at beginning of a launch is shown in Fig. 1. The free-fall height is measured from the water surface to the lowest point of the launching skid. The primary factors which affect the launch behavior of a free-fall lifeboat are its mass distribution, the length and angle of launch skid, and the free-fall height. These parameters interact to affect the orientation and velocity of the lifeboat at the time of water impact, the acceleration forces experienced by the occupants, the headway made by the lifeboat immediately after the water entry.

The complete launch of a free-fall lifeboat from the skid is composed of four phases, i.e., a) Sliding or ramp phase, b) Rotation restricted fall phases, c) Free-fall phases and d) Water entry phase,

As shown in Fig. 2. The ramp phase is that phase of the launch when the boat slides along the skid and ends when the center of gravity passes a certain point very close to the end of the skid. At that time the lifeboat begins to rotate and slides about the end of the skid. The rate of rotation increases until the boat is no longer in contact with the skid at which time the free-fall phase begins. The free-fall phase ends when the bow first contacts the water.

A fixed global coordinate system $(x, z)$ is set with $X$ axis describing an axis along the still water level and $Z$ axis corresponding to the vertical axis from the still water surface to the lowest end of the skid. A local coordinate system $(\xi, \zeta)$ is set with its origin at the center of gravity of the lifeboat at any time and instantaneous lifeboat axis direction $\xi$ axis. As shown in Fig. 1, both are right handed orthogonal Cartesian coordinate systems. The local coordinate system is used to describe the geometry of the lifeboat and to determine forces which act on the lifeboat. The global coordinate system is used to set the governing equations for time step simulation. The local coordinate system, which is fixed in relation to the lifeboat, translates and rotates with respect to the global coordinates system.

\section{a) Sliding Phase}

Sliding of boat begins when it is released and ends when the center of gravity $(G)$ is crossing a point close to the end of the launch skid. During this phase the lifeboat is constrained to slide along the skid, so it cannot rotate. The velocity of the lifeboat at the end of the launch skid is mostly dependent upon the length of the launch skid in front of the lifeboat, $L_{g o}$. The velocity increases as the distance $L_{g o}$, increases. The forces acting on the lifeboat during sliding are the gravity force $(M g)$, the normal reaction force $\left(F_{n}\right)$ between the skid and the rail, and the frictional force $\left(\mu F_{n}\right)$ between the skid and the rail. The governing equations are based on the global system which provides three degrees of freedom and are given as (Rahman, 2003): 


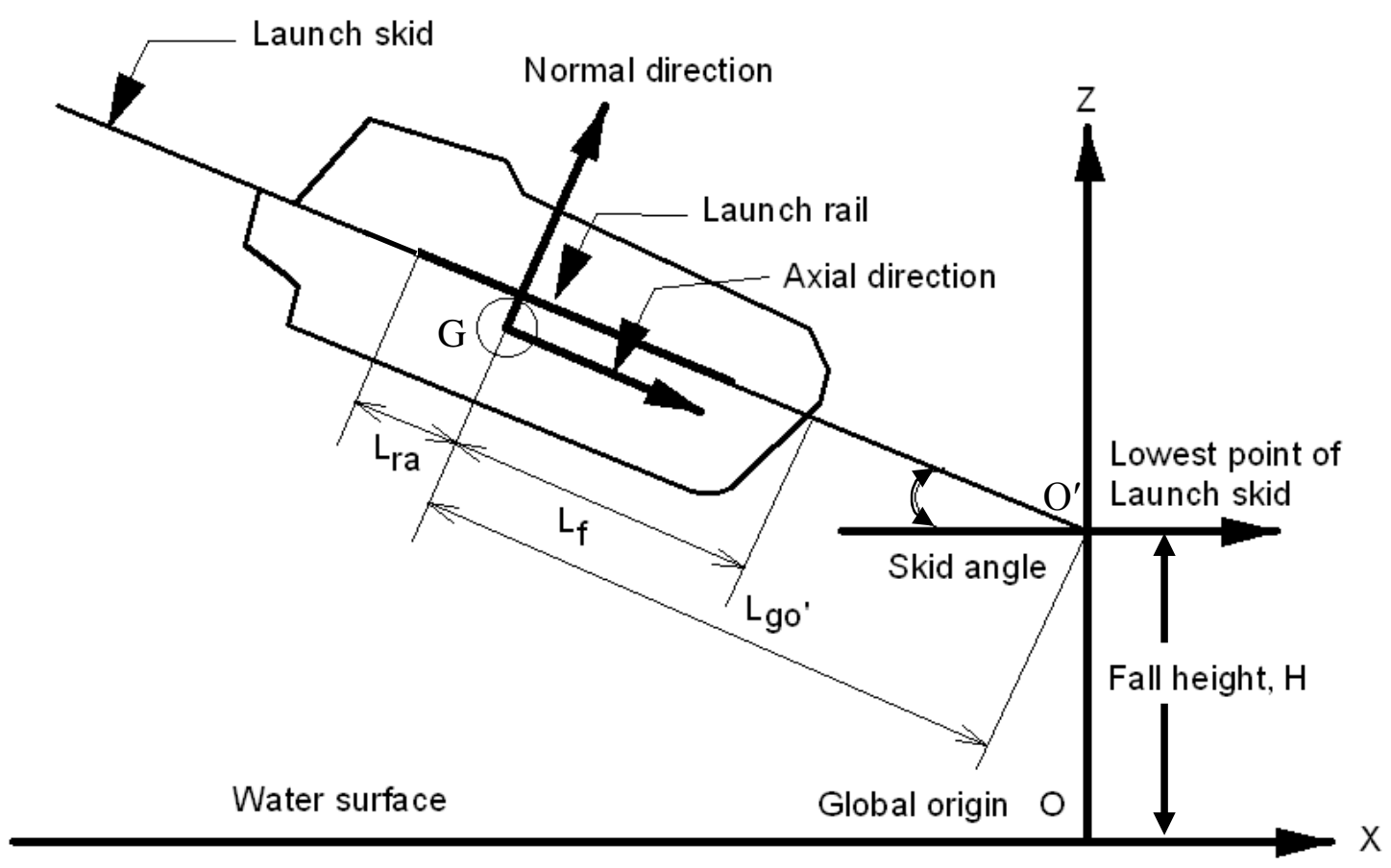

Fig. 1. Launch simulation of a free-fall lifeboat

$$
\begin{aligned}
& M \ddot{x}=F_{n}(\operatorname{Sin} \theta-\mu \operatorname{Cos} \theta) \\
& M \ddot{z}=F_{n}(\operatorname{Cos} \theta+\mu \operatorname{Sin} \theta)-M g \\
& I \ddot{\theta}=0
\end{aligned}
$$

In Eq. (1), $M$ is the mass of the lifeboat, $I$ is the rotational moment of inertia for pitching, $\mu=\operatorname{Tan} \phi$ is the friction factor and $\theta$ is the instantaneous angle of the axis of the boat with horizontal.

\section{b) Rotation Phase}

This rotation phase of the free-fall launch begins as the sliding ends and it continues until the boat is no longer in contact with the launch skid. The forces acting on the lifeboat during rotation are the gravity force $(M g)$, friction $\left(\mu F_{n}\right)$ parallel to the launch rail and a force normal to the rail $\left(F_{n}\right)$. A couple is produced by the weight of the boat and the reactive force on the launce rail, and it causes the boat to rotate as it slides off the launce skid. The equations of motions are:

$$
\begin{aligned}
& M \ddot{x}=F_{n} \operatorname{Sin}(\theta-\phi) / \operatorname{Cos} \phi \\
& M \ddot{z}=F_{n} \operatorname{Cos}(\theta-\phi) / \operatorname{Cos} \phi-M g \\
& I \ddot{\theta}=F_{n}\{x \operatorname{Cos}(\theta-\phi)-z \operatorname{Sin}(\theta-\phi)\} / \operatorname{Cos} \phi
\end{aligned}
$$

The launching parameters which affect the behavior of the lifeboat when it is rotating at the end of the skid are the distance between the center of gravity and the aft end of the launce rail $L_{r a}$, the angle from which the lifeboat is launched, and the velocity of the lifeboat when it begins to rotate. The angular momentum imparted to the lifeboat decreases as the distance $L_{g o}$, increases. This occurs because the velocity of the lifeboat at the beginning of the rotation phase increases as the distance to the end of the skid increases.

\section{c) Free-fall Phase}

The free-fall phase of the launch begins at the end of the rotation phase and continues until the boat touches the water surface. During free fall of the boat the only force acting on the boat is its gravity force $(M g)$ and hence the equations of motion are simple.

$M \ddot{x}=0 ; M \ddot{z}=-M g \quad ;$ and $I \ddot{\theta}=0$

Except for conditions with large fall heights and wind speeds, the influence of the air drag is insignificant. This influence results in only a decrease of vertical momentum due to gravity force. The horizontal and angular velocities of the boat remain almost constant. The rotation of the boat during the free-fall is very important as it determines the angle of attack at the water.

\section{d) Water Entry Phase}

The water entry of the free-fall lifeboat begins at the end of the free fall phase. During the water entry phase, the boat is acted upon by hydrostatic and hydrodynamic forces and the equations of motion can be given as:

$$
\begin{aligned}
M \ddot{x}= & F_{m n} \operatorname{Sin} \theta-F_{m a} \operatorname{Cos} \theta+F_{d n} \operatorname{Sin} \theta \\
& -F_{d a} \operatorname{Cos} \theta \\
M \ddot{z}= & F_{m n} \operatorname{Cos} \theta+F_{m a} \operatorname{Sin} \theta+F_{d n} \operatorname{Cos} \theta \\
& +F_{d a} \operatorname{Sin} \theta-M g+F_{b}
\end{aligned}
$$


$I \ddot{\theta}=-M_{m n}-M_{d n}-M_{b}$

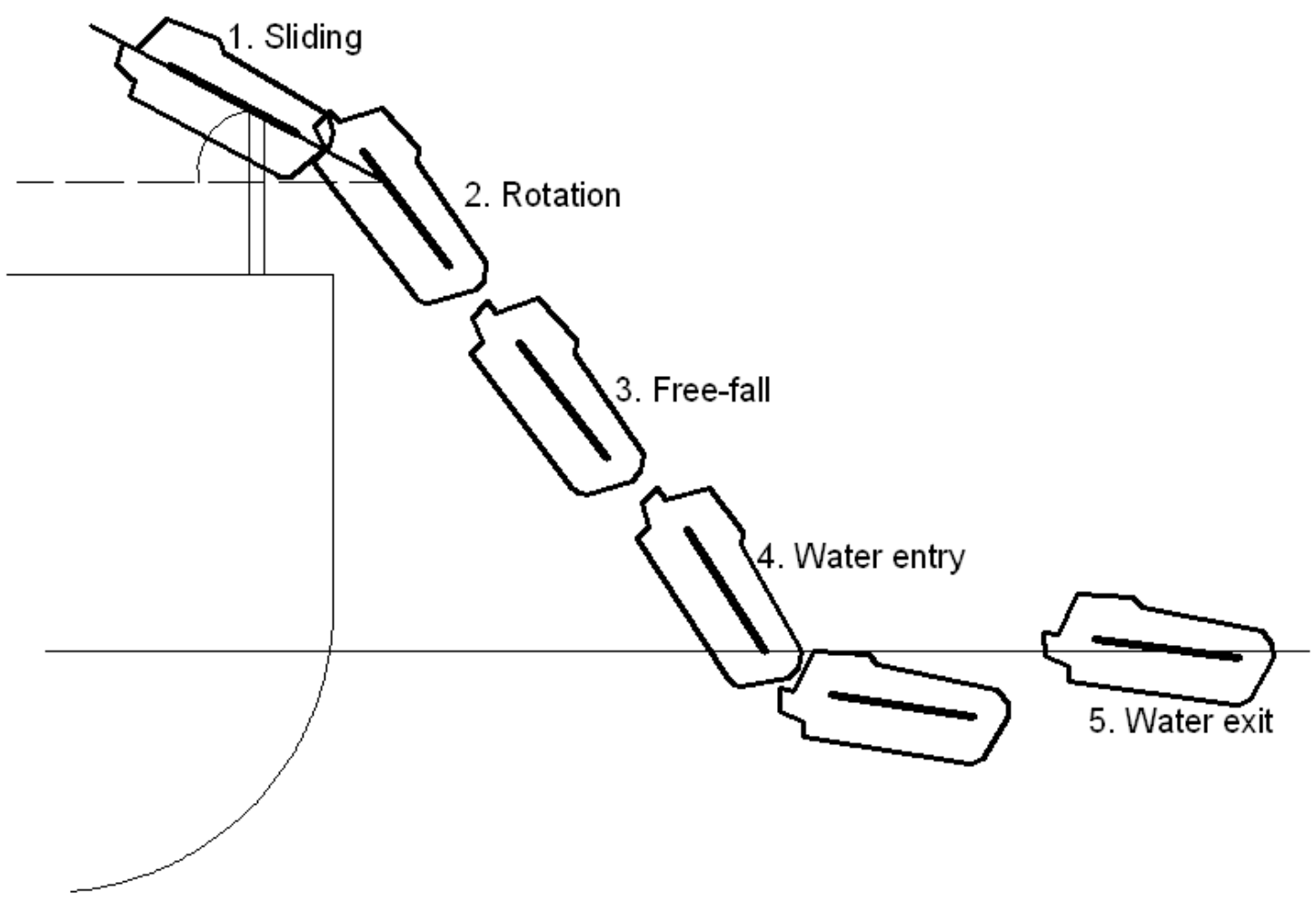

Fig. 2. Different phases of a free-fall lifeboat

Here, $F_{m n}$ is the force due to momentum transfer in the normal direction to the boat axis, $F_{m a}$ is the force due to momentum transfer in the axial direction, $F_{d n}$, normal drag force, $F_{d a}$ axial drag force, $F_{b}$, buoyancy force, $M g$, gravity force, $M_{m n}$, moment due to momentum transfer in the normal direction, $M_{d n}$, drag moment and $M_{b}$, buoyancy moment.

When the lifeboat first hit the water surface, high accelerations are experienced by the bow of the boat, this is bow impact. At that time, couple formed by the fluid forces and the weight of the lifeboat causes the angular momentum induced during the rotation phase to be reversed and the boat to return to even keel and this is stern impact.

Numerical integration of the equations of motions, as presented in this section, is performed by Newmark $\beta$ method (Press et al., 1987) to obtain velocity and displacement of the free-fall lifeboat from the computed accelerations.

\subsection{Hydrostatic and Hydrodynamic Forces and Moments}

The volumetric force, the normal drag force and the force due to momentum transfer in the normal direction have been calculated using a strip model where the forces per cross-section are calculated using the relative velocity and acceleration, and are integrated over the length of the falling boat. To perform the numerical integration, the boat has been discretized into forty segments in the longitudinal direction. The forces along the axis of the boat have been approximated in total. The buoyancy force is proportional to the immersed volume of the boat. This volume can be obtained by integrating the immersed cross-sectional area $A_{i}(\xi)$ along length of the boat. The total volumetric force and its moment around the center of gravity of the boat become:

$$
\begin{aligned}
& F_{b}=\rho g \int A_{i}(\xi) d \xi \\
& M_{b}=\rho g \operatorname{Cos} \theta \int A_{i}(\xi) \xi d \xi
\end{aligned}
$$

In Eq. (5), $\rho$ is the density of water, $\xi$ is the distance of a particular section from the center of gravity of the boat.

The hydrodynamic drag forces and moment in the local moving coordinate system have been computed using the following equations:

$$
\begin{aligned}
& F_{d a}=0.5 \rho C_{d a} A_{c m} V_{a x}\left|V_{a x}\right| \\
& F_{d n}=0.5 \rho C_{d a} \int(2 C) V_{n r}\left|V_{n r}\right| d \xi \\
& M_{d n}=0.5 \rho C_{d a} \int(2 C) V_{n r}\left|V_{n r}\right| \xi d \xi
\end{aligned}
$$

Where, $2 \mathrm{C}$ is the instantaneous wetted breadth of a particular section of the boat at a particular immersion, $A_{c m}$, is the cross-sectional area of the midship and $V_{a x}$ and $V_{n r}$ are the axial and normal velocity and these can be given by the following equations: 


$$
\begin{aligned}
& v_{n r}(\xi)=-\dot{x} \operatorname{Sin} \theta+(\dot{\gamma}-\dot{z}) \operatorname{Cos} \theta+\xi \dot{\theta} \\
& v_{a x}=\dot{x} \operatorname{Cos} \theta+(\dot{\gamma}-\dot{z}) \operatorname{Sin} \theta
\end{aligned}
$$

The drag forces in Eq. (7) are based on the cross-flow principle, which assumes that the incident flow can be split into orthogonal components, which are independent of each other. The axial drag coefficient $\left(C_{d a}\right)$ depends on both skin friction and end pressure whereas normal drag coefficient $\left(C_{d n}\right)$ is dominated by pressure drag. The drag coefficients for axial and normal flow have been chosen from Hoerner (1958).

The instantaneous position of the water surface due to wave can be computed by:

$$
\gamma(t)=\gamma_{a} \operatorname{Sin}(\omega t+\phi)
$$

where $\gamma_{a}, \omega$ and $\phi$ are the amplitude, angular velocity and phase angle of the wave respectively.

The wave starts at the time of release of the boat. When the phase angle is zero, the wave elevation is zero at this position and the elevation changes with the change of phase angle.

\subsection{Force and Moment due to Momentum Transfer in Normal Direction}

According to the von Karman (1929) momentum theory, when a body enters the water, its original momentum be distributed between the body and the surrounding. And the force acting on the body can be evaluated by the rate of change of momentum transfer to the surrounding water. The hydrodynamic force due to momentum transfer in the normal direction to the axis of the lifeboat has been formulated here on the basis of this momentum theory and assuming irreversible nature of the impact (Aanesland, 1987, Colwill and Ahilan, 1992). The force on an arbitrary cross-section at position $\xi$ with length $d \xi$ can be given as:

$$
d F_{m m}^{\prime}=\left[\frac{d}{d t}\left\{m(\xi, h) V_{n r}(\xi)\right\}\right] d \xi=\left[\frac{d m}{d t} V_{n r}+m \frac{d V_{n r}}{d t}\right] d \xi
$$

Where $d m / d t$, the time derivative of added mass $m$ ( $\xi$, $h$ ) for a particular section located at a distance $\xi$ from the center of gravity and having an immersion $h$, is

$$
\frac{d m}{d t}=\frac{\partial m}{\partial h} \frac{d h}{d t}
$$

But $d h / d t$ will be evaluated only when $V_{n r}>0$, which corresponds to conditions of increasing immersion. When $V_{n r}<0, d h / d t$ is set equal to zero. This treatment is based on considerations of momentum transfer only upon water entry and not during conditions associated with water exit.

The total force due to momentum transfer in normal direction can be obtained by integrating the force as described in Eq. (9a) throughout the length of the boat. The force and moment due to momentum transfer in normal direction around the center of gravity of the boat then become:

$$
\begin{aligned}
F_{m n}^{\prime} & =(-\dot{x} \operatorname{Sin} \theta-\dot{z} \operatorname{Cos} \theta) \int \frac{d m}{d t} d \xi+ \\
& \dot{\theta} \int \frac{d m}{d t} \xi d \xi+\ddot{\theta} \int m \xi d \xi-(\ddot{x} \operatorname{Sin} \theta \\
& \left.+\ddot{z} \operatorname{Cos} \theta+2 \dot{\theta} v_{a x}\right) \int m d \xi \\
M_{m n}^{\prime} & =(-\dot{x} \operatorname{Sin} \theta-\dot{z} \operatorname{Cos} \theta) \int \frac{d m}{d t} \xi d \xi+ \\
& \dot{\theta} \int \frac{d m}{d t} \xi^{2} d \xi+\ddot{\theta} \int m \xi^{2} d \xi-(\ddot{x} \operatorname{Sin} \theta \\
& \left.+\ddot{z} \operatorname{Cos} \theta+2 \dot{\theta} v_{a x}\right) \int m \xi d \xi
\end{aligned}
$$

During water entry, the bottom of the front part of the boat first hits the water surface with velocity $V_{a x}$ axially. At that time $V_{a x} \operatorname{Tan} \alpha$, the component due the axial velocity $\left(V_{a x}\right)$ and longitudinal bottom shape $(\operatorname{Tan} \alpha$, $\alpha$ is the instantaneous angle of the bottom with the axial direction) of the front part of the boat, adds some extra force in normal direction. This force causes rotation of the boat when it moves even with axial velocity only. Including this effect the equations for force and moment due to momentum transfer in the normal direction to be modified as:

$$
\begin{aligned}
& F_{m n}=F_{m n}^{\prime}+V_{a x} \int \frac{d m}{d t} \operatorname{Tan} \alpha d \xi \\
& M_{m n}=M_{m n}^{\prime}+V_{a x} \int \frac{d m}{d t} \text { Tan } \alpha \xi d \xi
\end{aligned}
$$

\subsection{Estimation of Sectional Added Mass}

von Karman approximated the added mass of a wedge shaped body as that of a flat plate with same length and width. Therefore, for a certain immersion the added mass of the water contained in a semi cylinder having the length equal to the length of the wedge and the diameter equal to the wetted width of the wedge at that immersion. Similar approximation can be applied for the free-fall lifeboat with proper modification. The boat has different shape for different sections, but it is possible to determine the wetted width at different immersion which in fact be used in this study to determine the added mass and impact force. Therefore, the added mass per unit length $m(\xi, h)$ in Eq. (11) and its derivative $\partial m / \partial h$ are functions of immersion $h(\xi)$ of the keel of the boat from the still water level. The immersion $h(\xi)$ can be expressed as:

$$
h(\xi)=\frac{(\gamma-z)+\xi \operatorname{Sin} \theta}{\operatorname{Cos} \theta}+h_{0}
$$

The distribution of the added mass $m(\xi, h)$ and its time derivative for different immersion have been evaluated according to the following relations:

$$
\begin{aligned}
& m(\xi, h)=\rho \pi C^{2}(\xi, h) / 2 \text { for } h(\xi)<d_{1} \\
& m(\xi, h)=\rho \pi C_{m}^{2}\left(\xi, d_{1}\right) / 2 \text { for } h(\xi) \geq d_{1}
\end{aligned}
$$


Where $C(\xi, h)$ is the instantaneous wetted half-width of a particular section at immersion $h$ and $C_{m}\left(\xi, d_{1}\right)$ is the maximum half-width at immersion $d_{l}$. The derivative $\partial$ $m / \partial h=0$ is used for $h(\xi)>d_{l}$ considering the flow separation from the body during water entry of the lifeboat.

Since different cross section have different shape and size, input data for those sections have been extracted from the body plan of the lifeboat model. The transverse section has been divided into a number of equally of distant ordinates and for the immersion between two consecutive ordinates; the wetted halfwidth is calculated by linear interpolation.

\subsection{Force due to Momentum Transfer in Axial Direction}

For the momentum transfer along the boat axis, similar approach has been adopted but the force has been approximated in total. The average acceleration (Rahman, 2003) between the bow and center of gravity has been used here. The force associated with the axial momentum transfer is:

$$
\begin{aligned}
F_{m a}= & \ddot{x} \operatorname{Cos} \theta+(\ddot{\gamma}-\ddot{z}) \operatorname{Sin} \theta+ \\
& \left.1 / 2 L_{f} \theta^{2}\right) m(l)+\frac{d m}{d l} \cdot \frac{d l}{d t} v_{a x}
\end{aligned}
$$

Here, $L_{f}$ is the distance between the fore peck and the center of gravity of the boat, $m(l)$ is the axial added mass and $d m / d l$ is its derivative, which depend on axial immersion $l$. Moreover, in Eq. (14), $d l / d t$ is set equal to zero when $V_{x a}<0$, i.e., the momentum transfer is evaluated only for advance of the boat and not for backward motion. The axial immersion $l$ and the distribution of the axial added mass $m(l)$ are:

$$
\begin{aligned}
& l=(\gamma-z) / \operatorname{Sin} \theta+L_{f} \\
& m(l)=m_{a x}\left\{2\left(\frac{l}{L}\right)^{3}-4\left(\frac{l}{L}\right)^{2}+\frac{5}{2}\left(\frac{l}{L}\right)\right\}
\end{aligned}
$$

for $I<L / 2$

and $m(l)=m_{a x} / 2$ for $I \geq L / 2$

The added mass distribution function is taken roughly from the change of the sectional area in the fore part of the boat, and $m_{a x}$, the axial added mass for full immersion, has been chosen on the basis of the added mass for an ellipsoid which is:

$$
m_{a x}=k \pi \rho L D^{2} / 6
$$

Where $L$ is the length of the boat, $D=\left(d_{1}+d_{2}\right)$ at midship of the boat and $k$ is a coefficient depending on the $L / D$ ratio.

According to the above approximation for axial immersion and added mass, the computed axial acceleration increases suddenly at the time the axis of the boat touches the water surface. However, the axial impact should occur as soon as the keel of the boat touches the water surface and gradually increases to the peak value. Therefore, a different added mass approximation has been used for this time interval between the touching of the keel and that of the boat axis to give a linear increase of the acceleration.

Now, substituting all these forces computed so far into Eq. (4), the horizontal, vertical and rotational motions of the boat in global coordinate system can be computed. However, an occupant on a free-fall lifeboat will experience the accelerations in local coordinate system. So the computed accelerations have been transformed into the local coordinate system according to the following relationships:

$$
\begin{aligned}
& a_{\xi}(\xi, \zeta)=\ddot{x} \operatorname{Cos} \theta-(\ddot{\gamma}+\ddot{z}+g) \operatorname{Sin} \theta-\zeta \theta+\xi \theta^{2} \\
& a_{\zeta}(\xi, \zeta)=\ddot{x} \operatorname{Sin} \theta+(\ddot{\gamma}+\ddot{z}+g) \operatorname{Cos} \theta+\xi \theta+\zeta \theta^{2}
\end{aligned}
$$

Where $a_{\xi}$ and $a_{\zeta}$ are the acceleration of the boat in the axial and normal direction.

\section{RESUlts AND Discussion}

The primary factors which affect the launch behavior of the FFLB are its mass distribution, lifeboat loading and initial condition of falling, e.g., the length and angle of launch skid, the length of guide rail after the centre of gravity and the free-fall height as shown in Fig. 1. These parameters interact to affect the orientation and velocity of the lifeboat at the time of water impact, the acceleration, the acceleration forces experienced by the occupants and headway made by lifeboat immediately after water entry.

A particular parent vessel has also a range of free-fall height depending on its loaded and unloaded conditions and there should have some limit of space for the skid also. Therefore it is necessary to investigate the motion and acceleration of FFLB for various loading conditions. These investigations are done by using a lifeboat model as described in Table 1. In this research the effect of different falling heights such as $H=1.5 \mathrm{~m}$, $1.75 \mathrm{~m}$ and $2.00 \mathrm{~m}$ on the behaviour of FFLB is studied.

Table 1: Principle dimensions of the lifeboat used in simulation

\begin{tabular}{|l|l|}
\hline Particulars of FFLB & Values \\
\hline Length $(\mathrm{m})$ & 1.00 \\
\hline Breadth $(\mathrm{m})$ & 0.358 \\
\hline Draught $(\mathrm{m})$ & 0.100 \\
\hline Displacement $(\mathrm{kg})$ & 15.30 \\
\hline$C_{m}$ at midship $(\mathrm{m})$ & 0.192 \\
\hline$d_{l}$ at midship $(\mathrm{m})$ & 0.211 \\
\hline$\left(d_{I}+d_{2}\right)$ at midship $(\mathrm{m})$ & 0.311 \\
\hline$K G(\mathrm{~m})$ & 0.181 \\
\hline$L_{r a}(\mathrm{~m})$ & 0.503 \\
\hline$G$ from midship $(\mathrm{m})$ & 0.003 \\
\hline Radius of gyration $(\mathrm{m})$ & 0.320 \\
\hline Scale & $1: 7.4$ \\
\hline
\end{tabular}




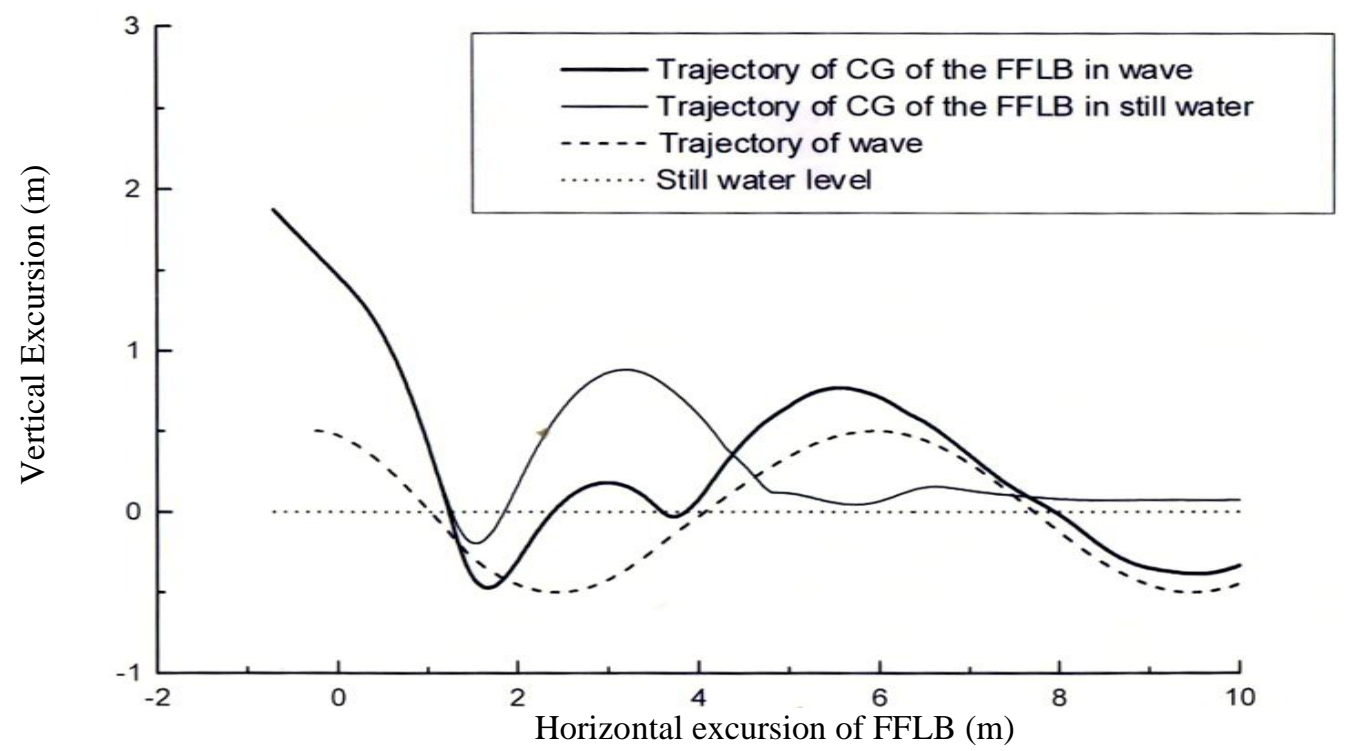

Fig. 3. Trajectory of CG of the FFLB in wave and in still water $\left(\theta=30^{\circ}, H=1.5 \mathrm{~m}, \gamma=0.5 \mathrm{~m}, T=2.0 \mathrm{sec}\right.$, $\left.\phi=0^{\circ}\right)$

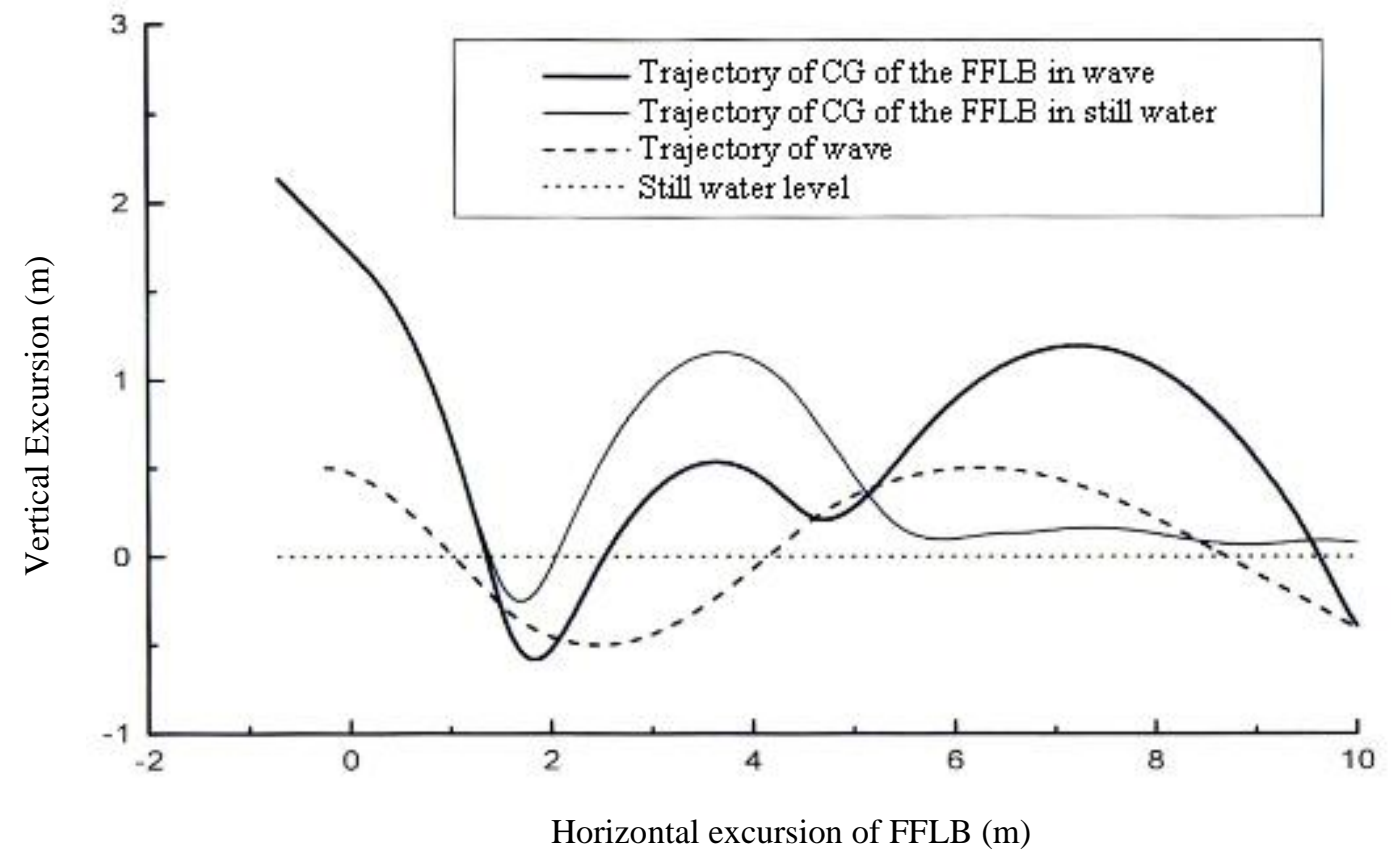

Fig. 4. Trajectory of CG of the FFLB in wave and in still water $\left(\theta=30^{\circ}, H=1.75, \gamma=0.5 \mathrm{~m}, T=2.0 \mathrm{sec}\right.$, $\phi=0^{\circ}$ )

\subsection{Trajectory Motion Analysis}

Figure 3 shows the trajectory of the center of gravity of the free-fall lifeboat in a wave of $0.5 \mathrm{~m}$ maximum amplitude, $2.00 \mathrm{sec}$. wave period and zero degree phase angle. The boat is falling from a height of $1.5 \mathrm{~m}$ with a falling angle of $30^{\circ}$. It slides along the skid of length $1.4 \mathrm{~m}$ with a sliding length $L_{g o}=0.80 \mathrm{~m}$. After that it rotates in clockwise direction and falls freely until it touches the water surface and then the impact force due to the change in added mass and buoyancy force start to act. Buoyancy force is increasing as long as the boat goes into water until it reaches to its maximum immersion $(0.5 \mathrm{~m})$. During this time, the boat starts rotating in the opposite direction (i.e., anti-clock wise) due to the buoyancy moment and the moment due to momentum transfer. At that time the axis of the lifeboat disappears fully inside water and a large buoyancy force pushes it up. 
M.M. Karim et al. / JAFM, Vol. 4, No. 1, pp. 77-88, 2011.

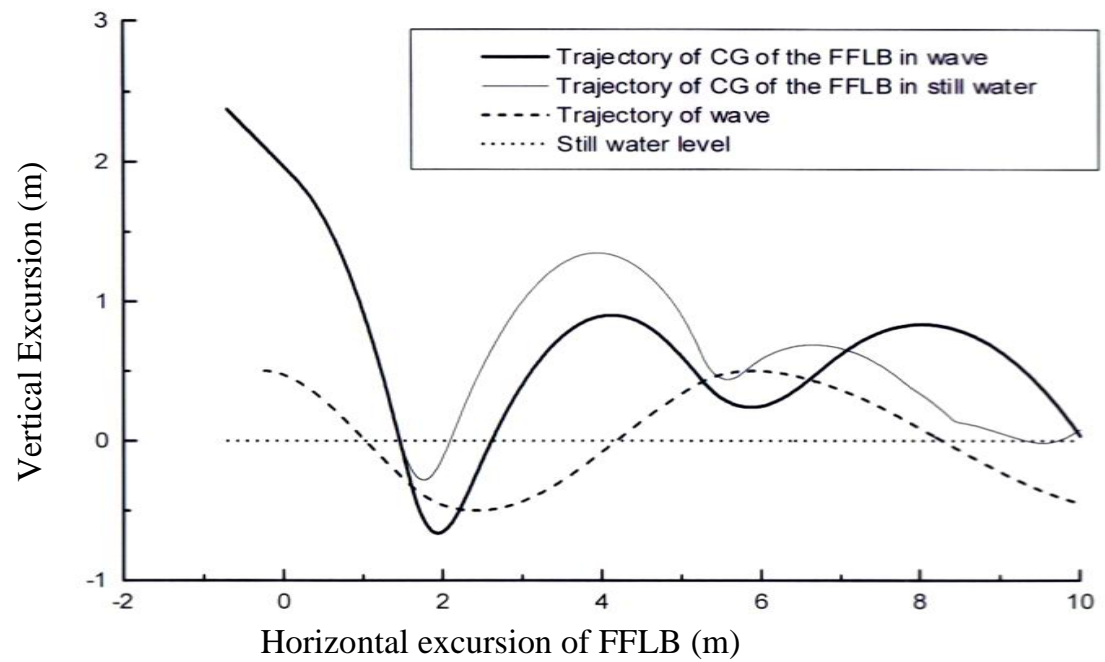

Fig. 5. Trajectory of CG of the FFLB in wave and in still water $\left(\theta=30^{\circ}, H=2.00, \gamma=0.5 \mathrm{~m}, T=2.0\right.$ sec, $\left.\phi=0^{\circ}\right)$

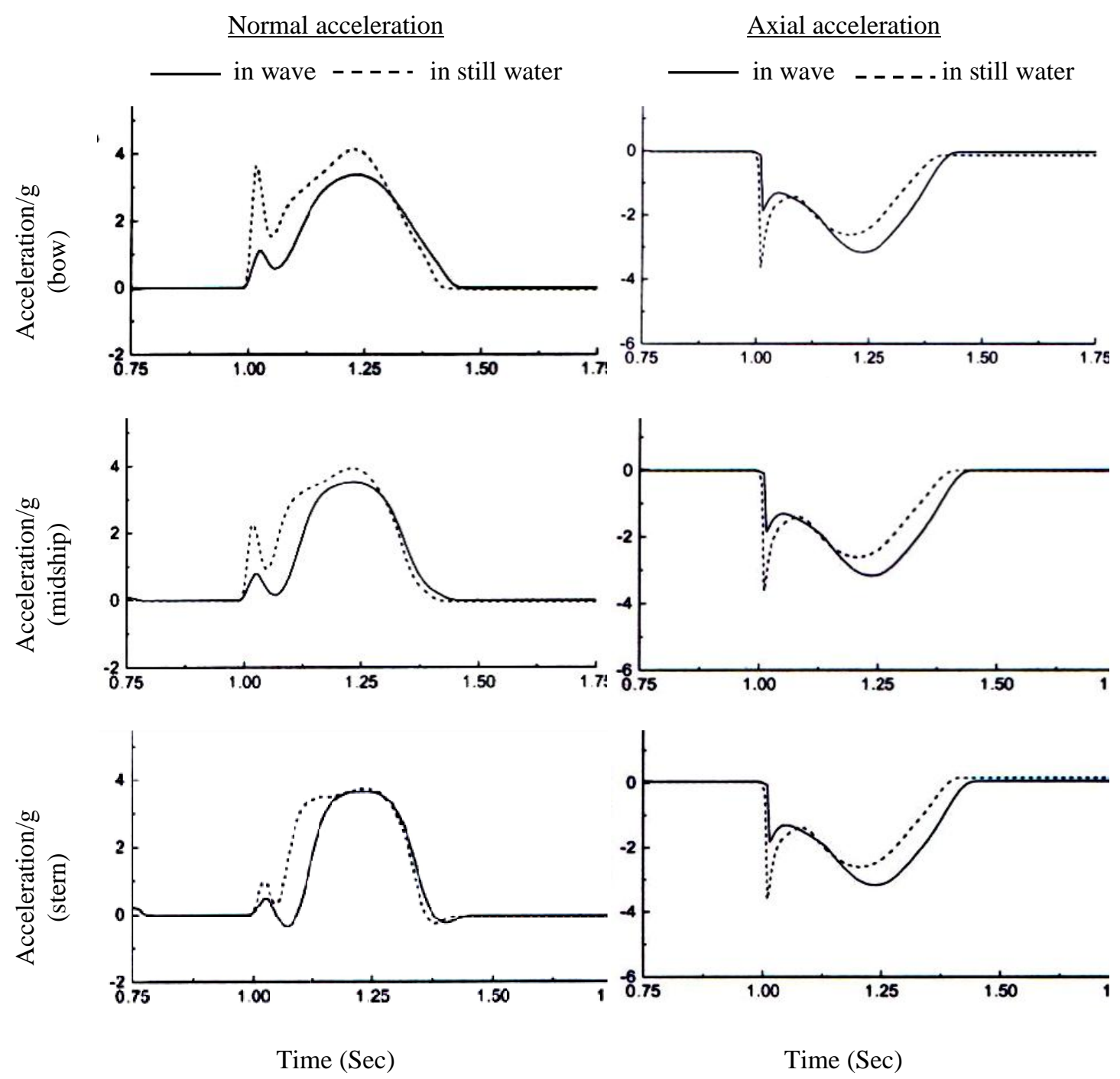

Fig. 6. Normal and axial acceleration of the boat at different positions in wave and in still water $\left(\theta=30^{\circ}, H=1.5, \gamma=0.5 \mathrm{~m}, T=2.0 \mathrm{sec}, \phi=0^{\circ}\right)$ 


\section{$\underline{\text { Normal acceleration }}$}
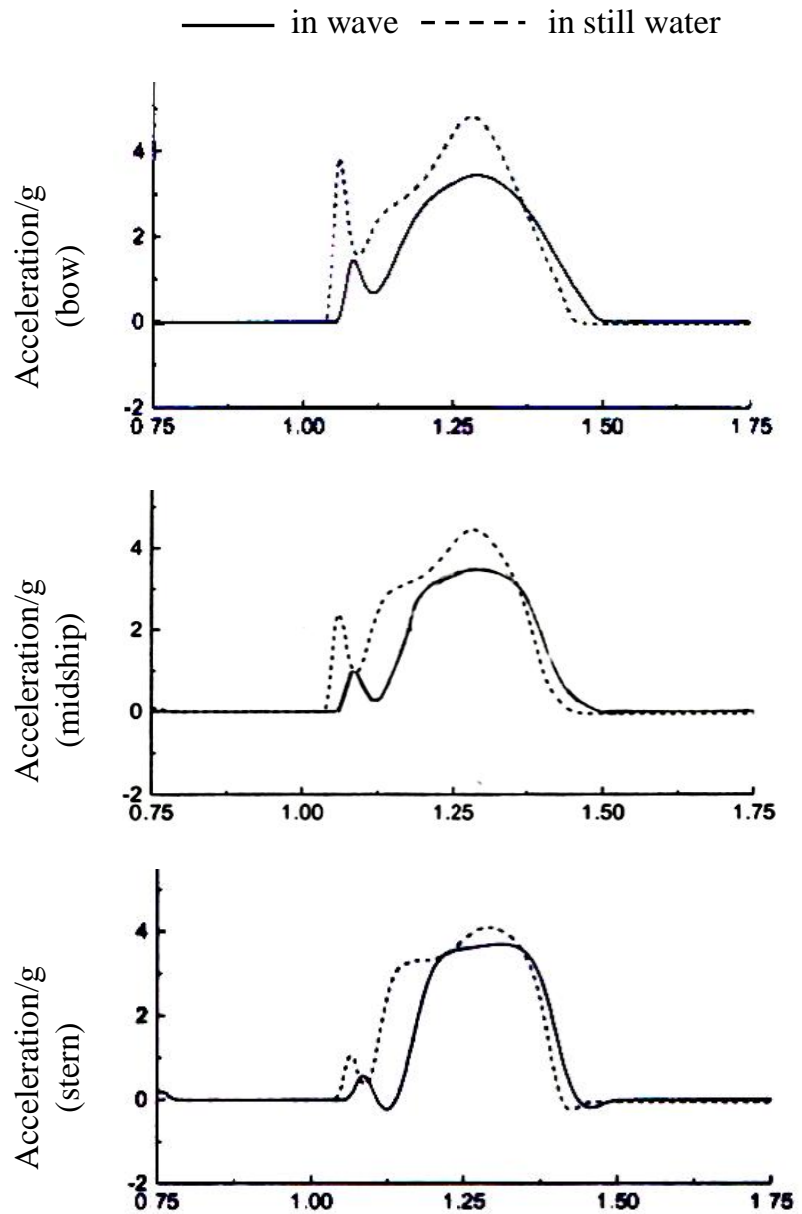

Time $(\mathrm{Sec})$ $\underline{\text { Axial acceleration }}$

in wave --- - in still water
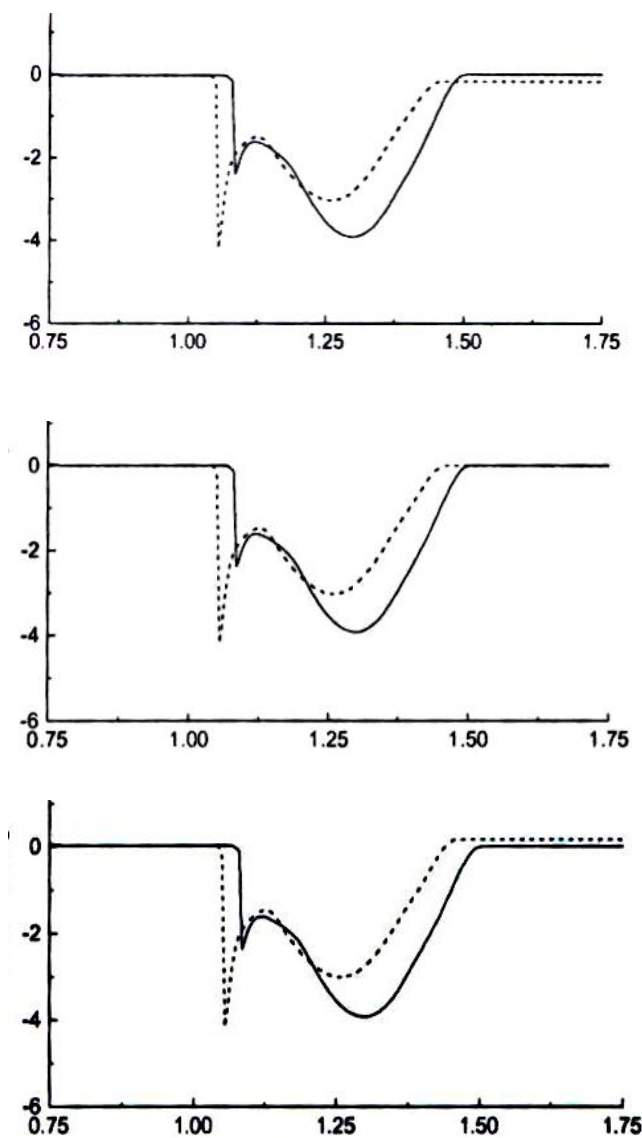

Time $(\mathrm{Sec})$

Fig. 7. Normal and axial acceleration of the boat at different positions in wave and in still water $\left(\theta=30^{\circ}\right.$,

$$
\left.H=1.75, \gamma=0.5 \mathrm{~m}, T=2.0 \mathrm{sec}, \phi=0^{\circ}\right)
$$

The boat then comes out of the water and falls down again into water with already forwarding some distance. Figs. 4 and 5 show the trajectory of motion of FFLB with falling height of $1.75 \mathrm{~m}$ and $2.00 \mathrm{~m}$ respectively. It is seen from the figures that maximum immersion of the boat increases as the falling height increases. The increase in falling height of the boat makes it to rotate more in anticlockwise direction causing a higher impact of the stern. This results in jumping of the boat to higher level of the first entry and also to move longer distance in the air before falling again into water. Due to the presence of wave, the boat is immersed again into water even at lower falling height.

\subsection{Analysis of Time History of Acceleration}

Figure 6 shows the computed acceleration in the normal direction of the axis of the boat at three positions (near bow, midship and stern) for the falling of boat into still water and in wave. The boat falls at an initial angle of $\theta=30^{\circ}$ with sliding length of $L_{g o^{\prime}}=0.80 \mathrm{~m}$ from falling height of $H=1.5 \mathrm{~m}$. It is seen from figure that at the bow position the peak acceleration in the normal direction caused by bow impact has the maximum value of nearly $3.4 \mathrm{~g}$. It is also seen that the stern impact occurs after the bow impact, the magnitude of the acceleration becomes comparatively large (approximately 3.6g) and the stern impact happens for a longer period. The peak acceleration due to bow impact in case of falling into still water is much higher than that in wave. This is due to large falling height. The value of acceleration due to stern impact is also higher up to peak value in case of falling in still water than that in wave.

The figure also shows the computed accelerations in axial direction of the boat at different positions. The peak decelerations by numerical simulation are greater than $3.5 \mathrm{~g}$ in three positions. 


\section{$\underline{\text { Normal acceleration }}$}
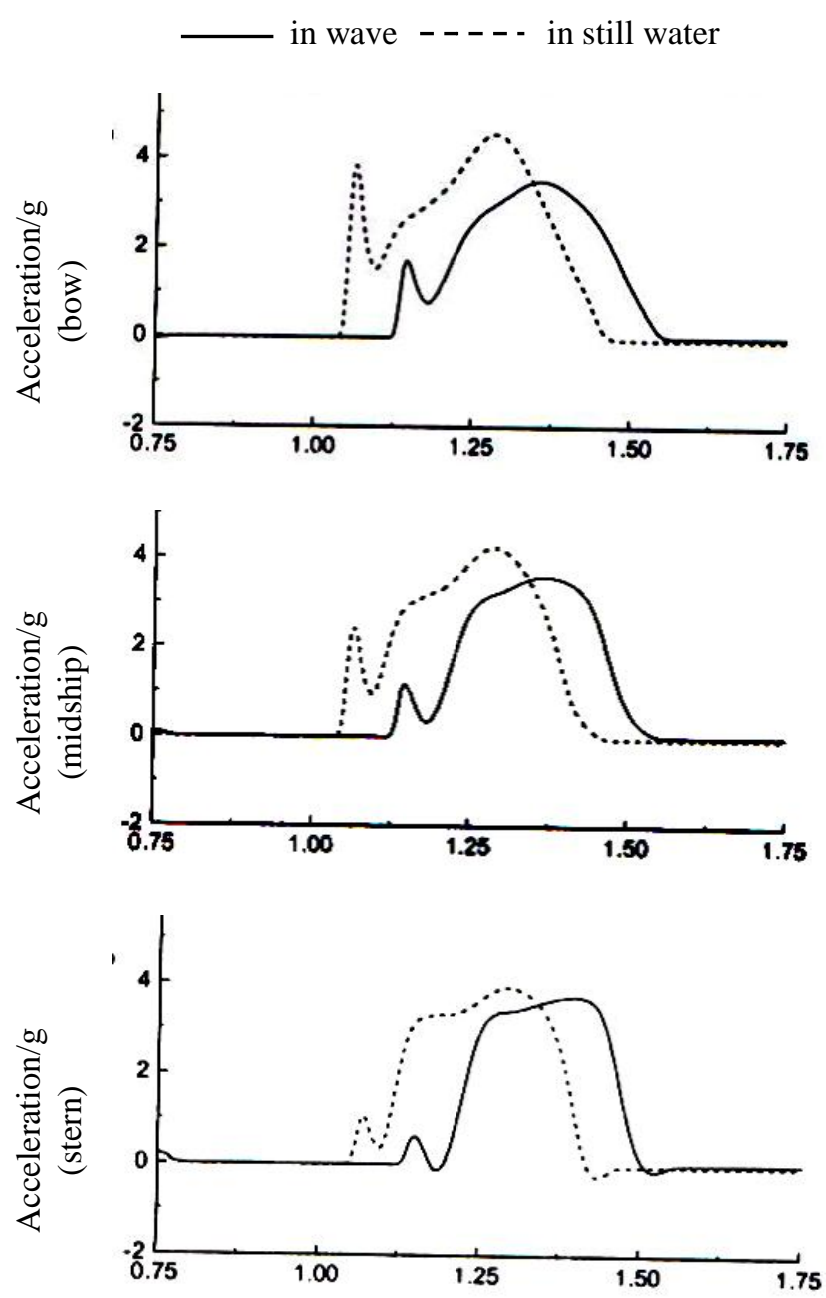

Time $(\mathrm{Sec})$ $\underline{\text { Axial acceleration }}$

in wave - - - - in still water
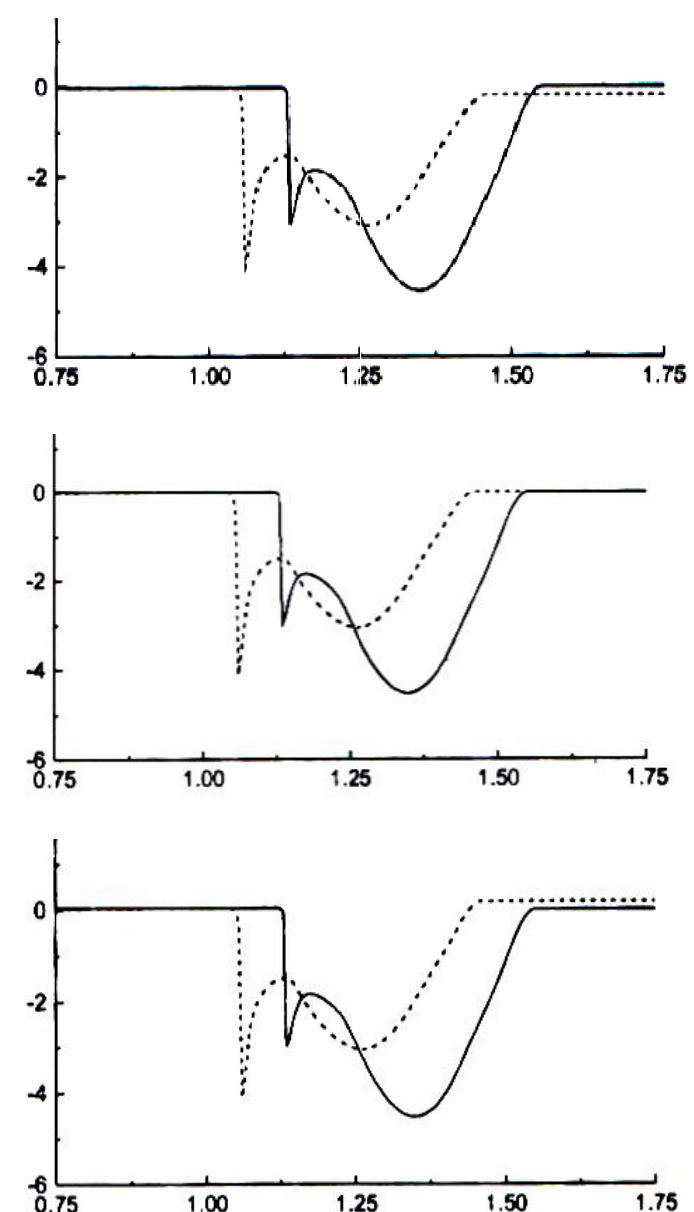

Time $(\mathrm{Sec})$

Fig. 8. Normal and axial acceleration of the boat at different positions in wave and in still water $\left(\theta=30^{\circ}\right.$,

$$
H=2.00, \gamma=0.5 \mathrm{~m}, T=2.0 \mathrm{sec}, \phi=0^{\circ} \text { ) }
$$

Figures 7 and 8 also show almost similar nature of curves of the normal and axial accelerations for falling heights $1.75 \mathrm{~m}$ and $2.00 \mathrm{~m}$ respectively. It is seen from figures that the acceleration both in normal and axial directions increases with the increase of effective falling heights. However, accelerations also increase when the boat hits into a rising water surface due to the addition of the effect of wave impact with the impact of boat. This increase in accelerations is harmful and causes discomfort to the occupants.

\subsection{Analysis of Hydrostatic and Hydrodynamic Forces}

The hydrostatic and hydrodynamic forces per unit weight in the normal direction of the lifeboat computed at the centre of gravity of the boat are shown in Fig. 9a. The numerical results have been obtained for falling angle of $\theta=30^{\circ}$, sliding length of $L_{g o}=0.80 \mathrm{~m}$ and falling height of $H=1.5 \mathrm{~m}$. In the time history of total force and force due to momentum transfer, contribution due to the latter is much higher than that of the former one, which is similar to the time history of normal acceleration at midship of the lifeboat. However, the magnitude of the maximum acceleration and nondimensional total force is different since in acceleration calculation, effect of added mass has also been taken into consideration. It is also seen from Fig. 9a that during water entry, only force due to moment transfer has very large contribution in total force and as the boat proceeds, the contribution of buoyancy increases. But contribution of drag force becomes significant as the boat immersed almost fully inside water (i.e., buoyancy is almost to its maximum) and decreases as long as the boat comes out of water. Therefore buoyancy and drag force control the motion of the lifeboat after full water entry. Contribution of gravity force in normal direction increases very slowly throughout its journey since the orientation of the boat changes. After coming out from water, the boat moves forward and already advanced 


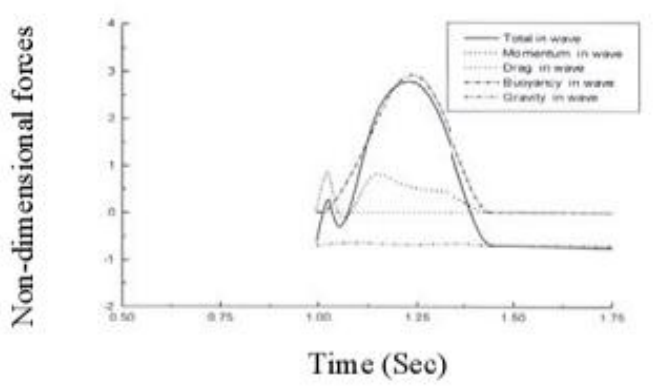

Fig. 9a. Non-dimensional forces at normal direction in wave $\left(\theta=30^{\circ}, H=1.5, \gamma=0.5 \mathrm{~m}\right.$, $\left.T=2.0 \mathrm{sec}, \phi=0^{\circ}\right)$

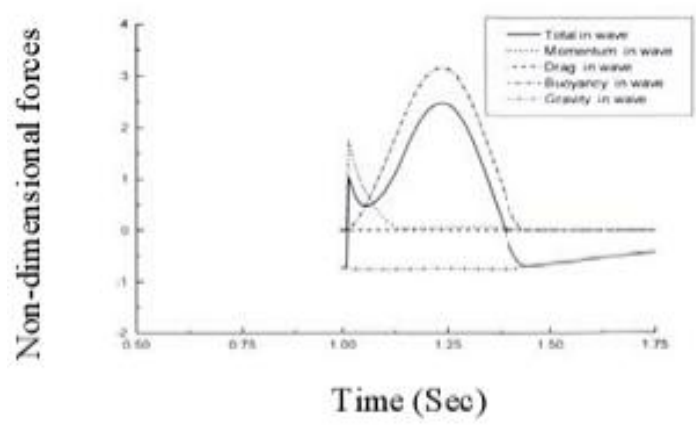

Fig. 9b. Non-dimensional forces at axial direction in wave $\left(\theta=30^{\circ}, H=1.5, \gamma=0.5 \mathrm{~m}, T=2.0 \mathrm{sec}\right.$,

$$
\left.\phi=0^{\circ}\right)
$$

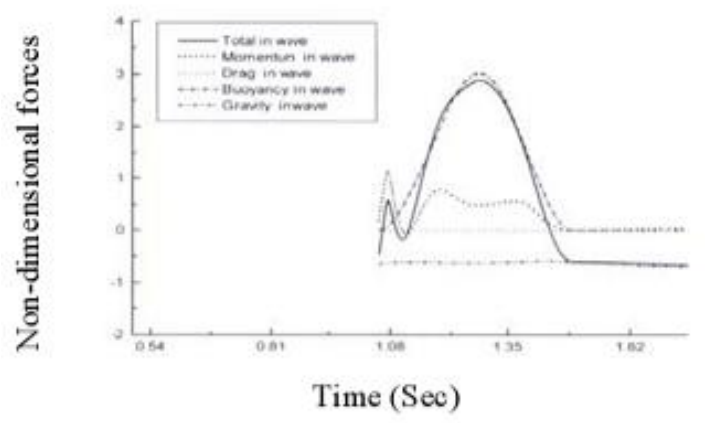

Fig. 10a. Non-dimensional forces at normal direction in wave $\left(\theta=30^{\circ}, H=1.75, \gamma=0.5 \mathrm{~m}\right.$,

$$
T=2.0 \mathrm{sec}, \phi=0^{\circ} \text { ) }
$$

a horizontal distance. It is to be noted that the force due to momentum transfer has the two portions: first of which depends on the added mass and acceleration and the second one depends on the rate of change of added mass and the velocity, i.e., impact force.

But for numerical simulation first portion has to be added with the generalized inertia force to get the vertical inertia force and hence only the impact has been shown here. Figure $9 \mathrm{~b}$ shows the time history of different non dimensional forces in axial direction for the same falling case. The contribution of impact in total forces is very significant during the water entry of the lifeboat as seen from the figure and the contribution of buoyancy increases as it proceeds inside water. The effect of drag force in axial direction is almost

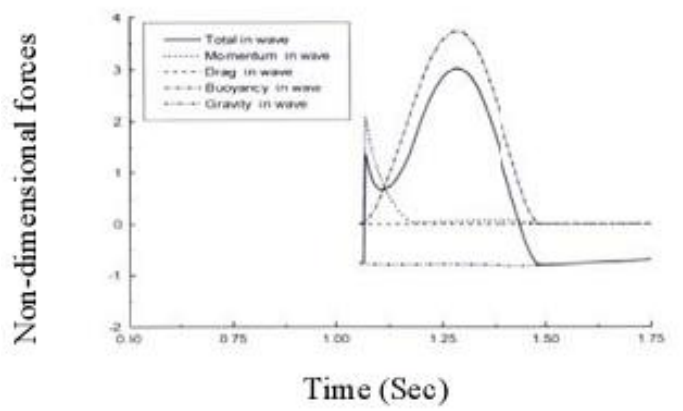

Fig. 10b. Non-dimensional forces at axial direction in wave $\left(\theta=30^{\circ}, H=1.75, \gamma=0.5 \mathrm{~m}\right.$, $T=2.0 \mathrm{sec}, \phi=0^{\circ}$ )

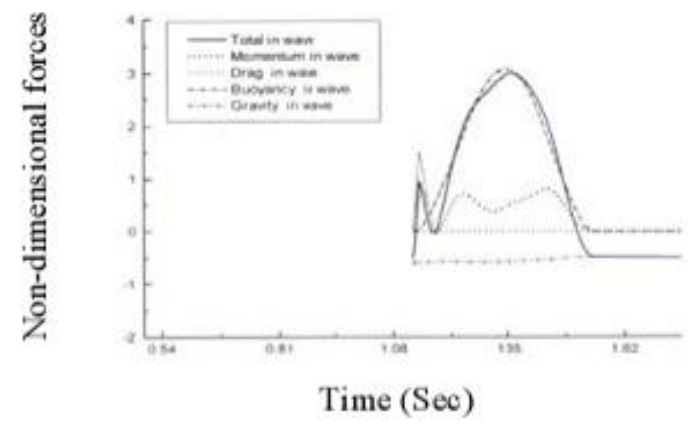

Fig. 11a. Non-dimensional forces at normal direction in wave $\left(\theta=30^{\circ}, H=2.0, \gamma=0.5 \mathrm{~m}\right.$, $T=2.0 \mathrm{sec}, \phi=0^{\circ}$ )
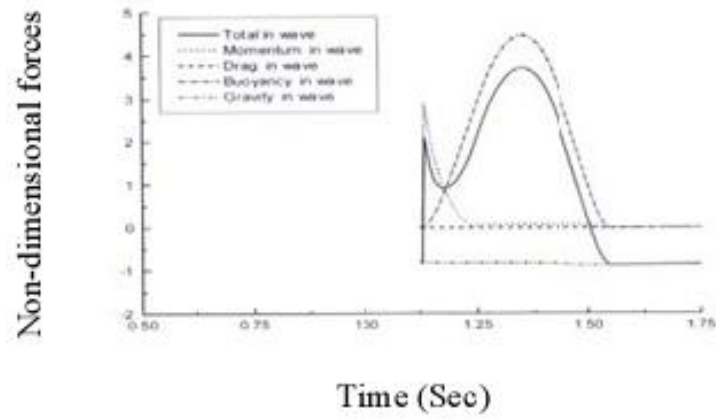

Fig. 11b. Non-dimensional forces at axial direction in wave $\left(\theta=30^{\circ}, H=2.00, \gamma=0.5 \mathrm{~m}\right.$,

$$
T=2.0 \mathrm{sec}, \phi=0^{\circ} \text { ) }
$$

negligible throughout the journey and effect of gravity decreases since the angle of axis of the boat with horizontal decreases. It is also seen that after full entry of the boat into water, only buoyancy controls the motion in axial direction. Figures 10 and 11 also show the normal and axial components of non-dimensional forces for falling height $1.75 \mathrm{~m}$ and $2.00 \mathrm{~m}$ respectively. It is seen from figures that as falling height increases, force components also increase.

\section{CONClusion}

The influence of falling height on the launch behavior of skid-launching free-fall lifeboat in regular waves is studied in this research. From the above mentioned study following conclusions can be drawn: 
1) The present mathematical model can be used to predict the magnitude and direction of the accelerations in different positions of a free-fall lifeboat. It can also be used to evaluate the motions of the boat for different falling heights.

2) As the effective falling height increases, the maximum immersion of the boat increases. The increase in falling height makes the boat to rotate more in anticlockwise direction causing a higher impact of the stern. This results in jumping of the boat to higher level of the first entry and also to move longer distances in the air before falling again into water.

3) The acceleration both in normal and axial directions increases with the increase of effective falling heights. However, accelerations also increase when the boat hits into a rising water surface due to the addition of the effect of wave impact with the impact of boat. The increase in acceleration causes harm and discomfort to the occupants.

4) Stern impact causes maximum acceleration both in normal and axial directions.

5) The hydrostatic and hydrodynamic force components in normal and axial direction increases with the increase of effective falling height.

\section{REFERENCES}

Aanesland, V. (1987). Numerical and Experimental Investigation of Accidentally Falling Drilling Pipes. Proceedings of OTC No. 5497.

Arai, M. and M.R.H. Khondoker (1994). Motion Simulation of Falling Bodies During Water Entry: Basic Study with Cylindrical Bodies. Journal of the Kansai Society of Naval Architects of Japan 222 .

Arai, M., M.R.H. Khondoker and Y. Inoue (1995). Water Entry Simulation of Free-fall Lifeboat (First Report: Analysis of Motion and Acceleration). Journal of the Society of Naval Architects of Japan 178.

Arai, M. and M.R.H. Khondoker (1996a). Prediction of the Performance of Free-fall Lifeboat Launching from Skid, Offshore Mechanics and Engineering Conferences. ASME paper no OMAE-96-430, Florence, Italy.

Arai, M., M.R.H. Khondoker and Y. Inoue (1996b). Water Entry Simulation of Free-fall Lifeboat (Second Report: Effects of Acceleration on the Occupants). Journal of the Society of Naval Architects of Japan 179.

Arai, M., M.R.H. Khondoker and Y. Inoue (1996c). Water Entry Simulation of Free-fall Lifeboat (Third Report: Evaluation of Effective Launching Parameters). Journal of the Society of Naval Architects of Japan 180.
Boef, W.J.C. (1992). Launch and impact of Free-fall Lifeboat (Part I and Part II). Ocean Engineering 19.

Brinkley, J.W. and J.T. Shaffer (1971). Dynamic Simulation Techniques for the Design of Escape System. Symposium of Biodynamic Models and Their Application, AMRL-TR-71-29.

Campbell, I. and A. Claughton (1983). Development of Lifeboat System for Offshore Rigs and Platforms. International Conf. on Marine Survival Craft, London.

Colwill, R.D. and R.V. Ahilan (1992, May). Reliability Analysis of the Behavior of Dropped Objects. Proceedings of OTC, Texas.

Hoerner, S.F. (1958). Fluid Dynamic Drag. New Jersey, USA.

Luo, Y. and J. Davis (1992). Motion Simulation and Hazard Assessment of Dropped Objects. Proceedings of ISOPE Conf. IV.

Muler, L. and H.G. Payer (1983). Loads and Strength of Free Fall Lifeboats. International Conf. on Marine Survival Craft, London.

Nelson, J.K., D.J. Fallon, T.J. Hirsch and J. Verhoef (1991). Mathematical Modeling of Free-fall Lifeboat Launch Behavior. OMAE Conf. 1-B.

Nelson, J.K., N.B. Regan, R. Khandpur, A.C. Landsburg and R.L. Markle (1994). Implementation of Free-fall Lifeboats on Ships. Marine Technology 31(4).

Nelson, J.K., D.J. Fallon, T.J. Hirsch and J. Verhoef (1995). Effects of CG Location on Free-fall Lifeboat Launch Behavior. Journal of OMAE 117.

Newman, J.N. (1997). Marine Hydrodynamics. the MIT Press, Cambridge, Massachusets.

Payne, P.R. (1981). The Vertical Impact of a Wedge on a Fluid. Ocean Engg. 8.

Press, W.H., B.P. Flannery, S.A. Teukolsky and W.T. Vetterling (1987). Numerical Recipes: The Art of Scientific Computing. Cambridge University Press.

Rahman, S.M.H. (2003). A Study on Behavior of Freefall Lifeboat in Regular Waves. M.Sc. Engg. Thesis, Dept. of Naval Architecture and Marine Engineering, BUET.

Shaar, C.M. and G.B. Bergman (1983). A Protective Water Entry Device for Free Fall Lifeboats. International Conf. on Marine Survival Craft, London.

Tasaki, R., A. Ogawa and Y. Tsukino (1990). Numerical Simulation and Its Application on the Falling Motion of Free-fall Lifeboats. Journal of the Society of Naval Architects of Japan 167.

von Karman, T. (1929). The Impact on Seaplane Floats During Landing, NACA T.N. 321. 